\title{
Gasoline New Timing and Flux Adjustable Rotary Valve Design (Hereinafter: Rotary Valve)
}

\author{
DU huiqi, DU dong \\ Tianjin Sino-German Vocational Technical College, Tianjin 300200
}

\begin{abstract}
Conventional gasoline engine with an umbrella valve control cylinder intake and exhaust, in order to achieve sealing effect, the valve is driven by the spring force; at the same time, when the cam opens the valve to overcome the spring force acting. Sealing the better, the more power consumed in the engine mechanical losses, the valve mechanism consumes about $30 \%$, which is not a small loss! This article describes a new type of rotary valve is to significantly reduce mechanical losses, so as to achieve energy saving purposes.
\end{abstract}

\section{Introduction}

At present, the gasoline engine is mainly used umbrella ventilation valve (see Fig. 1) controlled cylinder internal deflagration of the moment, the pressure is very high. If Mifengbuyan umbrella valve will leak, the piston will be reduced thrust, the engine efficiency is low. To allow the umbrella valve closing tightly, it requires a large spring force (see Fig. 2); Of course, in order to allow the valve to open the umbrella, with a cam drive link also large force, the more unwanted power consumption.

At present, the mechanical efficiency of the gasoline engine is about $35 \%$, meaning that only $35 \%$ of the energy is converted to useful work we need to rest for about $65 \%$ of energy are wasted as mechanical loss. The main manifestations of waste heat and mechanical friction do attack like. Mechanical friction loss accounts for 62$75 \%$ loss; loss of driving various accessories accounted for $10-20 \%$ of mechanical losses; driven mechanical supercharger loss accounts for 6-10\% loss; pumping loss accounts for $10-20 \%$ mechanical loss.

Umbrella valve mechanism and drive $30 \%$ mechanical loss. When mainly at low speeds, lubrication is in a critical state, the friction loss will significantly increase the proportion; and the load acting on the main valve spring force caused, integrated into the output shaft of the engine drag torque is most obvious at this time, accounting about $14 \%$ of the total energy $(65 \% * 75 \% *$ $30 \%=14 \%$ )! Five-valve four-cylinder gasoline engine, 20 gas valve opening and closing mechanical energy to be consumed can be imagined. To this end, we carried out a bold attempt to break through the traditional design concepts, the concept of the rotary valve.
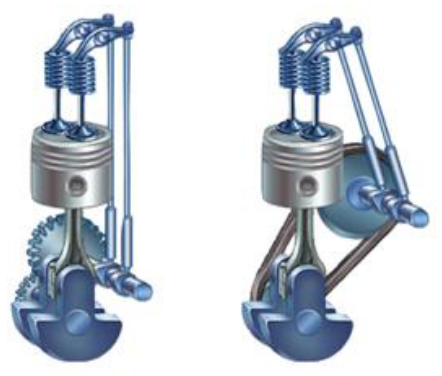

Camshaft

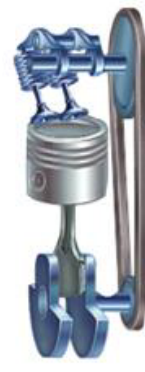

Camshaft

Figure 1. shaped valve.

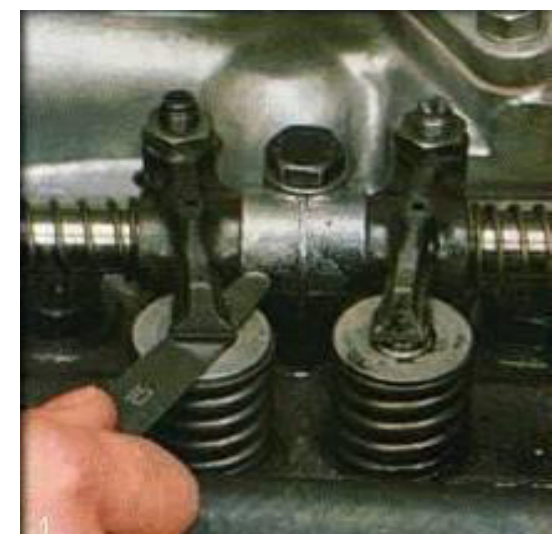

Figure 2. shaped valve.

\section{A historical causes gasoline umbrella valve}

Before the introduction of the new rotary valve concept, let's look at why the conventional gasoline engine is an umbrella organization of ventilation? We believe that the umbrella organization was based on the ventilation machine control level. Machinery and equipment from simple to complex, from single to comprehensively carry 
out development, with more and more complex machinery, the relevance of their actions, coordination is becoming a problem. At that time people can only continue to explore more specific and more complex mechanical mechanism along the mechanical structure design to meet the relevant mechanical and coordination of action. Such machinery is designed for mechanical drive mechanism and a mix of mechanical control mechanism, constitute the so-called complex machinery. Gasoline engine design also continue to use this method, but also a mix of mechanical drive mechanism and mechanical control mechanism. E.g., piston, connecting rod and crank mechanism is mechanically driven, controlling the opening and closing of the umbrella valve cams, connecting rods, levers, springs and other mechanical control means [1] (see Fig. 3,4).

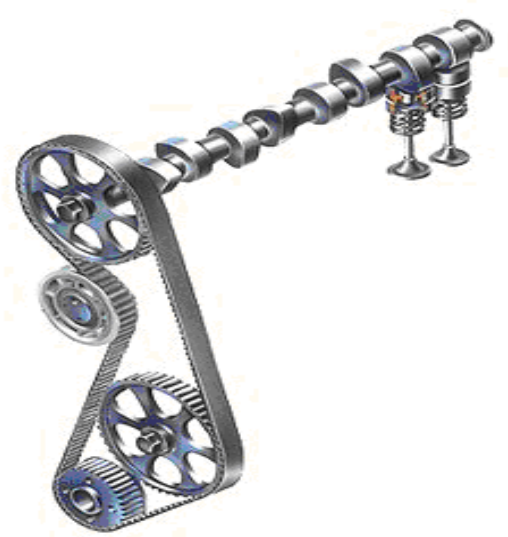

Figure 3. cam, connecting rod, lever.

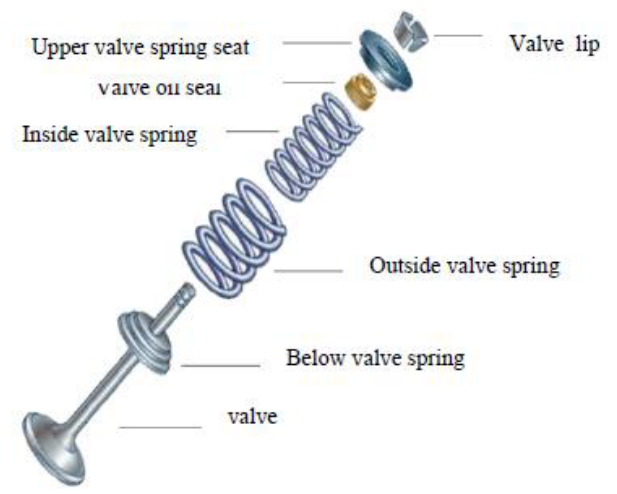

Figure 3. cam, connecting rod, lever.

At that time, electric drive and electronic control logic level limitations, most of the mechanical drive and control are provided by a driving source. Starting from a drive source, according to the control logic or timing requirements, mechanical drive and control mechanism to achieve the main driver of redistribution. This design concept can be seen everywhere in the traditional machinery, such as latitude typewriter pendulum control, the loom control, control, Shaper reciprocating mechanism inside the watch like a ratchet. Currently, these mechanical control mechanism and has been developed to a near-perfect situation. However, with increased levels of social development, the traditional mechanical design can not satisfy people more advanced automated machinery and equipment requirements, then the emergence of automated machinery and equipment mechatronics design. The following detailed description of this design concept used in the engine valve control.

\section{Gasoline rotary valve design ideas}

Faced with an increasingly complex machinery and equipment, the complex mechanical control mechanism becomes more and more bloated, but encountered a bottleneck for further development, then there is urgent need of a new control mechanism to replace the traditional mechanical control. Due to the rapid development of modern electronic control technology, we can now use the rotary encoder to replace the traditional mechanical cam mechanism, accurate and complete perspective of the angular velocity control. Decentralization drive element to the development of relations between the respective drive element action by an electronic control device such as a PLC and other coordination. Conventional gasoline engine intake and exhaust relationship with the spindle rotation is to rely on mechanical control mechanism, which does not meet modern mechatronics design. To make the gasoline engine become mechatronic devices, you must use the drive element decentralization principle, each intake and exhaust operation with a motor drive control, for eightvalve four-cylinder engine, eight little need to control the spindle motor coordinated action, complete with the best four-cylinder burst when suction pressure discharge valve.

Because the demand for gasoline engine performance improvement, such as combustion efficiency, energy saving, etc. the mechanical efficiency. Specifically, and puts forward some requirements of more and more precise control of angle and angular velocity, into the cylinder air intake time $t$, intake air quantity $\mathrm{Q}$, closing valve time $t$, ignition time $t$ fire, exhaust time $t$ row, row exhaust quantity $\mathrm{Q}$, these quantities are with the volume of three related, that is the shaft spindle position angle phi, angular velocity Omega shaft and spindle torque was axis. There is a definite link between them, in order to achieve the best control according to the need, to obtain the best output power, speed, acceleration, need to establish their function [2]. For example, P power $(\mathrm{kw})=$ torque $\mathrm{T}(\mathrm{nm})$ $\mathrm{x}$ speed omega (RPM) * 2 pi / 60, the simplified calculation become: $\mathrm{P}$ power $(\mathrm{kw})=$ torque $\mathrm{T}(\mathrm{nm}) \mathrm{x}$ speed $(\mathrm{RPM})$ /9549. Which torque $\mathrm{T}(\mathrm{nm})=\mathrm{f}(\mathrm{t}$ burning, into the $\mathrm{T}, \mathrm{Q}, \mathrm{t}$ ); speed $=\mathrm{f}$ (phi axis, Omega axis, as axis, $\mathrm{t}$ burn, into the $\mathrm{T}, \mathrm{Q}, \mathrm{t}$,). And according to the relevant variables of the function requirements of real-time adjustment. In order to adjust the real-time adjustment device, the traditional air intake and exhaust valve opening and closing time and the amount is fixed or there is a very adjusted, it is difficult to meet the requirements of the above functions for extreme operation requirements. Therefore, it is necessary to design a can meet the realtime adjustment of the device and the valve action time and action quantity can be stepless adjustment, realize the spindle angular position diameter shaft, spindle angular velocity Omega axis and spindle resistance torque was axis function. 


\section{Gasoline rotary valve structure and how it works}

Based on the above analysis, design a device to complete this function is running relationship, which is the main problem to be solved herein. Conventional internal combustion engine intake and exhaust valve is completed by an umbrella, which the valve is closed by spring force to achieve, in order to prevent the umbrella valve leakage, we must use a larger spring force mushroom valve and air inlet in close contact, the valve opening by the cam against the spring force of the inlet valve and the umbrella separately. Especially in the five-valve fourcylinder gasoline engine, 20 gas valve opening and closing mechanical energy to be consumed is considerable, accounting for about ten percent of the mechanical energy output [3].

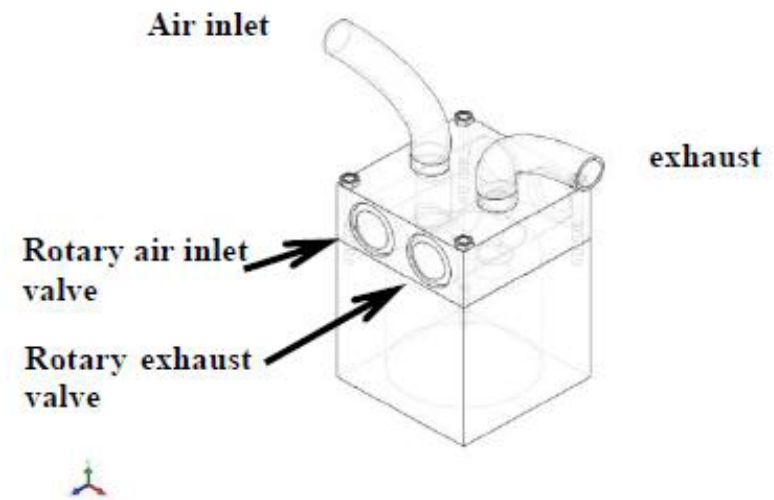

Figure 4. new shape of valve.

To overcome the umbrella valve mechanical energy consumption, we have designed a new type of valve, its shape shown in Figure 3, which changed the traditional mushroom-valve structure, replaced by a rotary valve,the structure is open on a cylindrical spool a cylindrical spool perpendicular to the axis of the through-holes. In the body also has a corresponding diameter of the airway, the valve spool is rotated 90 degrees, you can complete the valve opening and closing operation, namely the valve opening and closing is done by rotating the spool. Since the rotary valve is completed by sealing the structure itself, and can resist the instantaneous deflagration tremendous pressure required to overcome the additional spring, so reducing the valve opening and closing operation of the energy consumption.

Internal combustion engine is a sophisticated machine, various mechanical motion mechanism of an organic whole, used to be on mechanical contact the various agencies, between each driven mechanical institutions now have no fixed mechanical mechanism, is to rely on a kind of invisible things connect each drive, they are single chip microcomputer, PLC and other logical control unit, to coordinate the relationship between various actions between the drive, which is driving the decentralized design concept. Rotary valve implementation method is described below.

Rotary valve valve core rotation is by small step motor or servo motor drive, because the motor is vector control motor, control motor pulse output terminal Q0.0 issued by PLC, the pulse frequency is up to $20 \mathrm{KHZ}$, if choose motor subdivision control pulse number is $10000 /$ $\mathrm{r}$, Angle precision can reach $360^{\circ} / 10000 \mathrm{p}=0.036^{\circ} / 1$ $\mathrm{p}$, namely each pulse can make the motor around $0.036^{\circ}$. If the opening of $90^{\circ}$ and $0.036^{\circ}$ is open for this $0.036^{\circ}$ $90^{\circ} /=0.0004$ times. So, in terms of internal combustion engine valve control, the accuracy is high, the Angle and speed can be precisely controlled. In fact, open unnecessarily high precision requirement, can choose motor subdivision control pulse number is $400 / \mathrm{r}$, under the condition of the pulse frequency of $20 \mathrm{KHZ}$, speed $=$ $20000 / 400=50 \mathrm{r} / \mathrm{s}=3000 \mathrm{r} / \mathrm{min}$. Due to the rotation gas every turn $180^{\circ}$ open gate valve core, so the rotation of the $3000 \mathrm{r} / \mathrm{min} 6000 \mathrm{r} / \mathrm{min}$ of internal combustion engine valve support. Rotary valve when opening or closing, is with the spindle angular position and angular velocity and torque at that time. Angular position information acquisition is to rely on and principal axis coaxial rotary encoder, it can rotate in a week, to launch 36000 pulse, namely each pulse represents $0.1^{\circ}$. Angular velocity is calculated, by the number of pulses through calculating unit of time, such as computer read 100 pulses within 0.1 seconds, rather read 1 seconds 1000 pulses angular velocity omega $=1000^{\circ} / \mathrm{s}$. According to the same method can also get angular acceleration, and then according to the angular acceleration can get the output torque and power. Of course, also can be used to linear motor rotating valve adjusted by the amount of the valve core to open, adjusted according to the mathematical model, the internal combustion engine achieve ideal running status.

In the hardware set up is completed, according to the theory of fuel combustion and the actual operating experience, in the PLC program can be easily write control program. For example, the main shaft turn to any Angle, make a revolving valve motor start or stop. In the actual debugging engine, can be easily in the program by rewriting the pulse number value, to the best control state. Of course also have control of the fuel delivery, air door and oxygen, etc. In a word, through the above control method, can complete accurate control, convenient for the internal combustion engine at full combustion, and reduce the fuel consumption and environmental protection.

\section{Technical innovations is mainly manifested in two aspects of hardware and software of the five points, below shows its advantages respectively}

Using rotating valve control of internal combustion engine intake and exhaust Mechanical structure of the rotating valve itself ensures that can easily turn, open breath consumed less mechanical energy, and can be multi-layer type $\mathrm{O}$ sealing ring to prevent the leakage of high pressure gas.

rotating valve with step motor or servo motor drive Rotary valve of the valve core with stepper motor or servo motor drive directly, belong to this kind of motor 
vector control model, can accurately control the motor shaft Angle, especially suitable for rotary valve drive.

Rotating valve opening area size can adjust Rotary valve open area size can be adjusted with step motor or servo motor, so that you can under the condition of different speed setting different cross-sectional area, and as the output control functions, through the PLC control of the motor, the adjustment of the complete circulation.

Flux valve opening and closing time and open closed digital; Valve open and close and cross-section of the valve is controlled by step motor or servo motor, the motor is belong to the vector control of motor, the motor stator coil magnetic field is produced digital vector synthesis of a rotating magnetic field, magnetic field and the Angle of the rotating magnetic field can accurately adjust speed, it's digital provides the basis for internal combustion engine rotating valve control, intelligent control created the conditions for internal combustion engine [4].

Control valve opening because of the stepper motor or servo motor motor belongs to the vector control motor, the controller is based on PC control from the PLC pulse sequence. So from the pulse number and pulse frequency can be operating conditions such as omega axis, Phi axis, as axis, burning $\mathrm{T}, \mathrm{t}, \mathrm{Q}, \mathrm{t}$ function. The mathematical model is established in the combustion laboratory experimental data and experience for people to grasp, with PLC programming language, and constantly improve and optimize.

\section{Concluding remarks}

This paper presents a new type of internal combustion engine valve, which can accurately control the time and the flow of air intake and exhaust. The valve and the traditional umbrella valve to reduce driving the power consumed by the valve, to realize the digital and intelligent control, improve the combustion quality, reduce pollution emissions, there is a very high popularization value.

\section{References}

1. Chen Jiarui, Automobile structure,(Beijing: Mechanical Industry Press ,2009)

2. Wang Wangyu,Automobile design(Beijing: Mechanical Industry Press, 2011)

3. Zhang Jilong,Development status and trend of optimization technology of gas distribution mechanism. Mechanical engineer 11, 83-84 (2015)

4. Chen Xinquan,The design principle and development of variable valve system from VVT-i to VVTL-i and cam control technology: equipment manufacturing technology 10, 104-106 (2008) 